\title{
A Study of the Streets and Alleys of Traditional Settlements in Taihang Mountains - Taking Wangjinzhuang, Shexian County as an Example
}

\author{
Wei Su${ }^{*}$, Yalei Liu² \\ ${ }^{1}$ School of Architecture and Design, Hebei Polytechnic Institute, Shijiazhuang 050091, Hebei Province, China \\ ${ }^{2}$ Hebei Jiuyizhuang Chen Technology (Group) Co., Ltd., Shijiazhuang 050035, Hebei Province, China \\ *Corresponding author: Wei Su, 609591943@qq.com
}

Copyright: () 2022 Author(s). This is an open-access article distributed under the terms of the Creative Commons Attribution License (CC BY 4.0), permitting distribution and reproduction in any medium, provided the original work is cited.

\begin{abstract}
Wangjinzhuang in Shexian County, which has a long history, is a representative of the traditional settlements in Taihang Mountains. Through the in-depth study of the morphological characteristics of Wangjinzhuang settlement, this paper fully excavates its value as a traditional settlement. The overall pattern of the settlement form is complete, showing a linear layout form; the road system is graded, and the structure is clear; the spatial form shows a combination of points, lines, and planes; public buildings are distributed in an orderly manner, with clear functions, and they have a high degree of research value.
\end{abstract}

Keywords: Traditional settlement; Street space; Wangjinzhuang

Online publication: March 4, 2022

\section{Overview of the settlement}

Wangjinzhuang, which belongs to Jingdian Town, is located deep in the mountains of Taihang, approximately five kilometers away from Shexian County. The village has five administrative villages with 4,417 people. After the 1950s, through the efforts of the villagers, the entire village has been comprehensively managed, making the current Wangjinzhuang seemingly brand new.

Wangjinzhuang settlement gradually developed and expanded, but the construction of the village improved after the development of Qing Dynasty. Its streets, ancestral halls, temples, and residential buildings, all formed a system; its residential buildings also had a fixed pattern. The development and expansion at that time were planned, so the whole layout and spatial texture of the settlement remained the same.

The connection between residential buildings and public buildings in Wangjinzhuang settlement is mainly the utilization of streets and public spaces. Public buildings are reasonably distributed in the settlement and assume corresponding functions, while residential buildings are enriched in the settlement under the framework of the road system and the distribution of public buildings, which together constitute the entire settlement, as shown in Figure 1. 


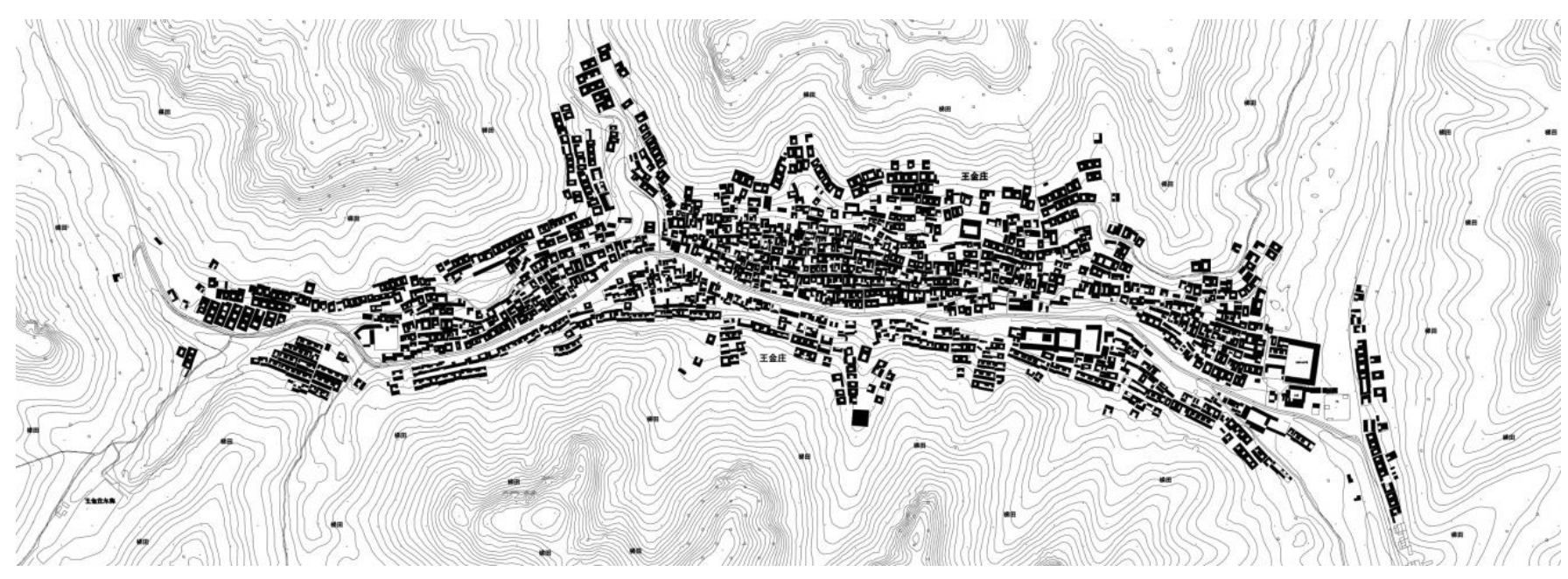

Figure 1. Wangjinzhuang settlement

On the north side of the main street, the buildings are densely distributed with abundant sunshine and a stone-slab street with unique space. Wangjinzhuang settlement gradually developed and expanded. The buildings are covered with mountains on both sides of the valley. The continuous extension to both ends of the main street will cause a deviation from the center, which is not easy to manage. Therefore, the linear layout of Wangjinzhuang is divided into five sections.

\section{The street space of Wangjinzhuang settlement}

\subsection{The evolution of its street space}

In the changes of settlements, the traces of time are preserved in the spatial morphological characteristics of the settlements ${ }^{[1]}$. There are two modes of evolution of streets and alleys: (1) streets first, and then houses; streets are formed through the comprehensive effect of rational order and feng shui principles; (2) houses first, and then streets; this is formed through the development of natural geographical environment. Wangjinzhuang neither follows the evolution mode of streets and alleys followed by houses, nor the evolution mode of houses followed by streets and alleys, but with the increase of residential buildings, it is naturally enclosed and has both spirits of rationality and irrationality.

The buildings on both sides of the main street are the first level of the settlement (Figure 2). As the population grew, the scale of the settlement expanded. The lanes extended to both sides and the buildings on both sides form the second level of the settlement (Figure 3). The architectural style, material, and structure of the roads on the first level are relatively uniform, whereas the roadways extending upward on the second level are more diversified, with different lengths, twists, and turns. After long-term development and evolution, the road network of the current Wangjinzhuang settlement is formed. Wangjinzhuang's road classification is shown in Figure 4. 


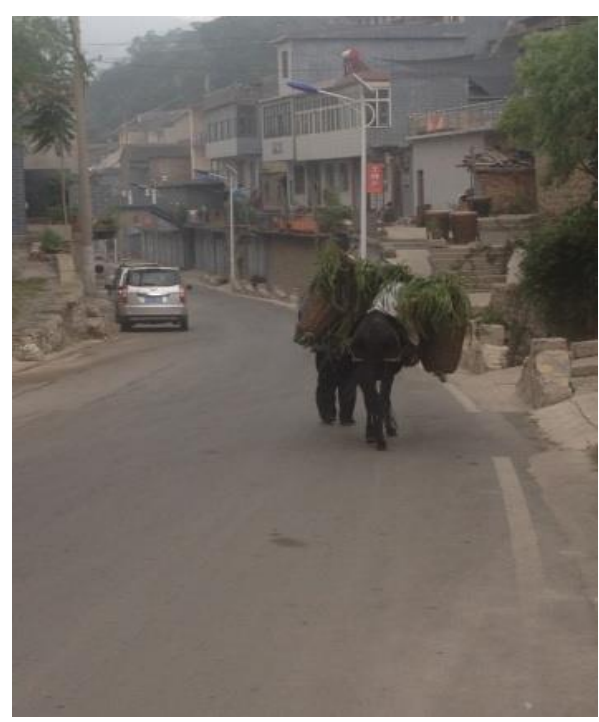

Figure 2. First-level street

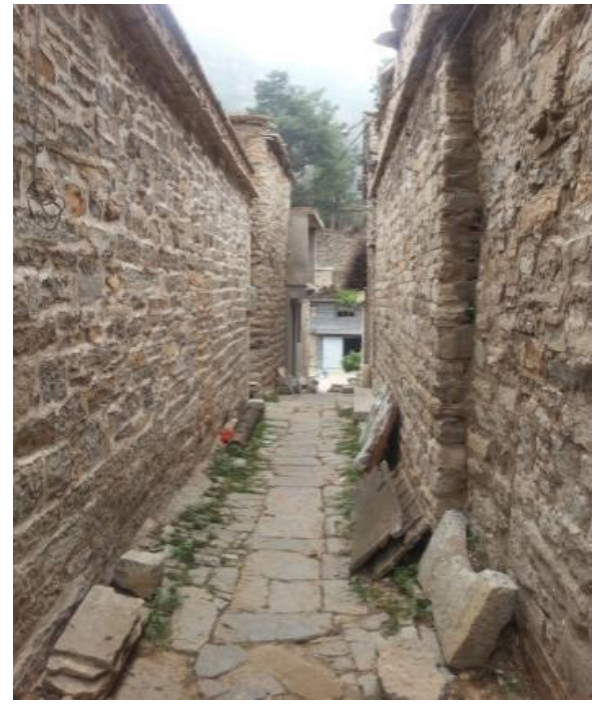

Figure 3. Second-level roadway

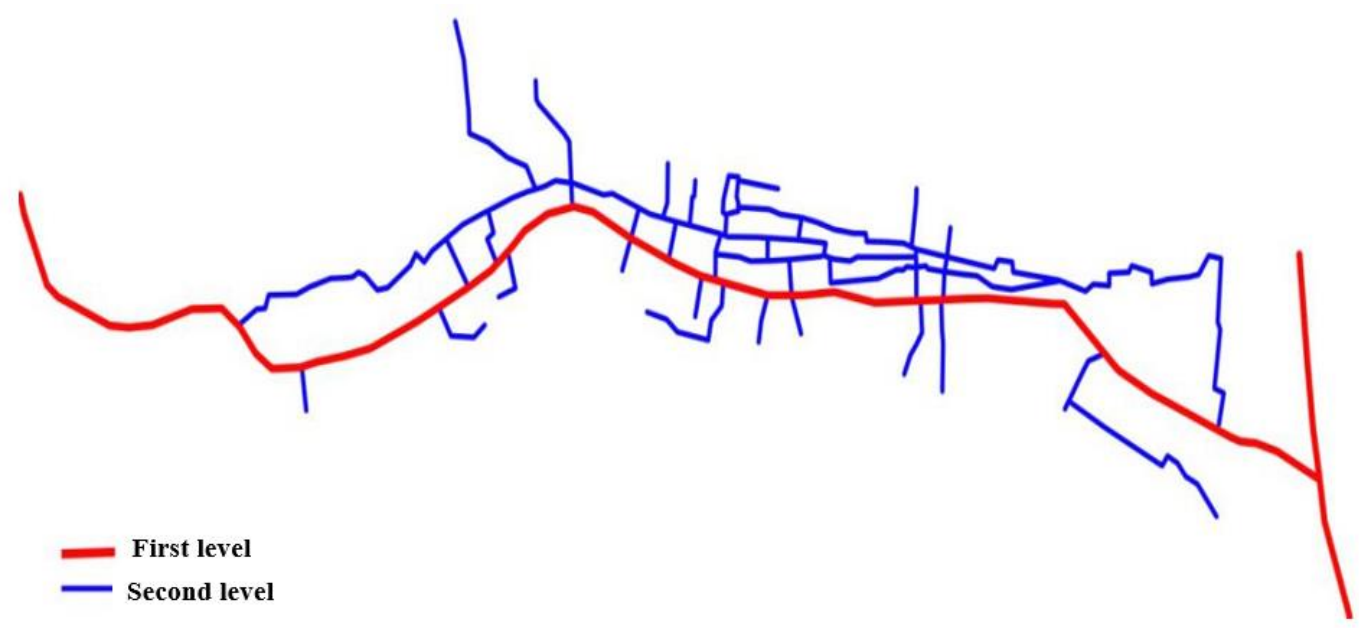

Figure 4. Wangjinzhuang's road classification

\subsection{Spatial composition of its streets}

The road system of Wangjinzhuang settlement consists of two levels. The first level is the main street that runs through the east and west of the settlement. The road is wide enough for motor vehicles to travel. The second level comprise of lanes connecting the main street and the buildings, leading to buildings on both sides of the main street. These dense lanes are organically cross-connected with the main street, forming the road network of the entire village.

The main street is 3.5-5 meters wide, with a height-to-width ratio of 1:3-1:2. This is related to the location and topography of the settlement. The main street runs through the entire settlement in the eastwest direction, winding along the mountain, forming the horizontal axis of the settlement, and dividing it into two parts: north and south.

The roadways connect the courtyards and the main street. Streets and alleys are the traffic space for the residents, and they are also the stage of life ${ }^{[2]}$. Their multiple functions ensue a changing and orderly form. The roadways in Wangjinzhuang can be divided into two types: traffic roadways and living roadways. Traffic roadways meet the daily traffic use of villagers, while living roadways directly connect courtyard spaces and can be used as public spaces. Most of the traffic lanes are relatively narrow, with width of less than 1 meter. The living roadways are access roadways for the villagers, in which the height-width ratio is relatively comfortable, and the interface is flexible and rich. 


\subsection{Space nodes of its streets and alleys}

The streets and alleys of Wangjinzhuang settlement are neatly distributed, forming a fishbone-shaped street and road system. The spatial nodes of its roads include intersections of streets and alleys, courtyard entrance spaces, and public square spaces.

(1) Street intersection space

Streets and alleys are not simple straight lines, but the intersection of multiple lines, thus forming a multilevel road network system. The intersection of streets and alleys generate nodes. The intersection nodes of streets and alleys in Wangjinzhuang have three forms: T-shaped, Y-shaped, and intersections, among which T-shaped intersections are the main ones. Wangjinzhuang settlement is undulating and has distinct layers. Based on the three types of intersections, various types of intersection nodes with height differences are formed.

(2) Courtyard entrance space

The main constituent element of the settlement is the courtyard space, and the entrance of the courtyard becomes the transition space connecting the inside and outside of the courtyard ${ }^{[3]}$. The courtyard entrance space belongs to the street and alley, but it also belongs to the courtyard. Therefore, it has a dual nature of being public and private. When the entrance space of the courtyard is used as a public space, it is the main place for villagers to come together. Gathering in front of the door is one of the leisure activities of the villagers. The front part of the courtyard also provides a place for recreation.

(3) Public space

Based on the village records, the original development point of Wangjinzhuang was the Liu Family Courtyard (including the South Courtyard and the North Courtyard). It is the main activity place of the villagers. In settlements, most of the places for people to take part in social activities appear with the formation of public buildings, such as ancestral halls, temples, and theaters. Most of the public spaces in settlements are naturally formed. They are mostly open spaces in front of iconic buildings and are shaped irregularly, with relatively random spatial layout. The ancestral hall of Wangjinzhuang is located at the center of the settlement, and it is an important space for people to come together. After long-term development and planning, the open space in front of the ancestral hall has become more regular in shape, forming a small rectangular space.

\section{The spatial form of Wangjinzhuang settlement}

Wangjinzhuang is surrounded by mountains. There are artificial forests and wild forests on the surrounding hillsides. The natural ecology is relatively intact. Walking in it is like walking in a "green world." Vibrant residential buildings can be seen in this greenery; the terraced fields integrated with the mountains are scattered, and the crops are colorful, changing along with the four seasons.

\subsection{Point-shaped space}

Point-shaped space is the basic element that constitutes the settlement space. Point-shaped space includes circles, triangles, squares, polygons, and irregular shapes. The point-shaped space here refers to landscape elements, such as stone towers and water wells, in Wangjinzhuang settlement and the elements formed by them.

\subsection{Linear-shaped space}

Linear-shaped space mainly refers to the layout structure of streets and alleys. The terrain of the settlement is complex, and the heights are scattered. Therefore, the shape of the streets and alleys also twists and turns according to the terrain, extending from the main street to both sides and forming a fishbone-like network structure. The main street is the widest in the settlement. The trend of the street is parallel to the contour 
line. Alleys are generally distributed perpendicular to each other and function to connect residential areas and the main street.

The features of linear-shaped space in Wangjinzhuang are as follows: the scale and height-width ratio of streets and alleys of different grades have different spatial characteristics; the turning point is large, which makes people look forward to the scenery after that.

\subsection{Plane-shaped space}

Plane-shaped space is an important modeling element in settlement space, which includes regular polygon and irregular polygon ${ }^{[4]}$. The plane-shaped space in Wangjinzhuang settlement is generally a public open space, including the village entrance square, the open space enclosed by the ancestral hall, the Liu family compound, and the square in front of the theater.

\section{Analysis of the public buildings in Wangjinzhuang settlement}

Residential buildings in settlements are living spaces for families, while public buildings are public spaces for collective activities, which are important parts of the settlement space. The public buildings in Wangjinzhuang provide residents with space for different purposes and play an irreplaceable role in their lives. Public buildings of different natures have different architectural features, enriching the overall pattern of the settlement.

The most typical public buildings in Wangjinzhuang are the ancestral hall, the stage, and the temple. These buildings provide an irreplaceable public place for social interaction in isolated and closed villages. They are also a concentrated reflection of local folk customs, architectural techniques, and artistic levels.

\section{Conclusion}

The composition of the settlement not only contains the physical environment, but also the material carrier of the residents' social life. The residential houses, streets, and public buildings in a settlement are the key elements that make up the order of the settlement, and they directly affect the morphological layout of the settlement. The linear overall layout structure of Wangjinzhuang creates a fishbone-shaped street system and a spatial structure combining points, lines, and planes. With its superior location along with rich historical and cultural resources, Wangjinzhuang has gradually formed a unique settlement.

\section{Funding}

Hebei Province Human Resources and Social Security Research Project (JRS-2021-3248).

\section{Disclosure statement}

The authors declare no conflict of interest.

\section{References}

[1] Tian K, Chen Y, 2021, Changes and Spatial Characteristics of Traditional Settlements in the Enyang Section of Micang Ancient Road. China Famous City, 35(12): 68-74. DOI: 10.19924/j.cnki.16744144.2021.12.010

[2] Zhou Z, Li H, Jiang W, 2021, Research on the Spatial Form of Traditional Settlement in Anyuan Village, Chongren County, Fuzhou City. Architecture and Culture, 2021(11): 230-231. DOI: 10.19875/j.cnki.jzywh.2021.11.086 
[3] Li Y, 2012, Research on the Traditional Settlements and Dwellings of Liujiazhai in Shexian County, Hebei University of Engineering, Handan.

[4] Wang H, Fu X, Hou Q, 2021, Study on the Streets and Lanes of Traditional Settlements in Ancient Huizhou. Journal of Xi' an University of Architecture and Technology (Social Science Edition), 40(03): 34-41. DOI: $10.15986 / \mathrm{j} .1008-7192.2021 .03 .006$

\section{Publisher's note}

Bio-Byword Scientific Publishing remains neutral with regard to jurisdictional claims in published maps and institutional affiliations. 